\title{
Kubo spins in nanoscale aluminum grains: A muon spin relaxation study
}

\author{
Nimrod Bachar $\odot,{ }^{1, *}$ Aviad Levy, ${ }^{2}$ Thomas Prokscha $\odot,{ }^{3}$ Andreas Suter, ${ }^{3}$ Elvezio Morenzoni, ${ }^{3}$ \\ Zaher Salman $\odot,{ }^{3}$ and Guy Deutscher ${ }^{2}$ \\ ${ }^{1}$ Department of Quantum Matter Physics, University of Geneva, CH-1211 Geneva 4, Switzerland \\ ${ }^{2}$ Raymond and Beverly Sackler School of Physics and Astronomy, Tel Aviv University, Tel Aviv 69978, Israel \\ ${ }^{3}$ Laboratory for Muon Spin Spectroscopy, Paul Scherrer Institute, 5232 Villigen PSI, Switzerland
}

(Received 25 February 2018; revised manuscript received 19 December 2019; published 27 January 2020)

\begin{abstract}
We report muon spin relaxation rate measurements on films composed of aluminum grains having a size of a few nm, with a large energy level splitting of the order of $100 \mathrm{~K}$. The films range from weakly metallic to insulating. In the insulating case, the low-temperature relaxation rate is consistent with the presence of single electron spins in grains having an odd number of electrons, known as Kubo spins. The relaxation rate temperature dependence follows an activation law having an energy scale in agreement with the average level splitting. In weakly metallic films, inter-grain junction spins may contribute substantially to the smaller relaxation rate.
\end{abstract}

DOI: 10.1103/PhysRevB.101.024424

\section{INTRODUCTION}

Several years ago, Kubo [1] predicted that small metallic particles having an odd number of electrons must bear an inherent magnetic moment because of the unpaired electron at the highest occupied energy level. The Kubo spin can be observed under two conditions. First of all, the thermal energy $k_{B} T$ must be small compared to the energy level splitting $\delta E$. For a particle radius $R$ of $1 \mathrm{~nm}$ this splitting is of the order of $100 \mathrm{~K}$. Second, charge fluctuations in the particles must be restricted, as hopping of an electron in and out will erase the Kubo spin effect. To maintain charge localization in an ensemble of particles, the electrostatic charging energy $e^{2} / 2 R$ must be much larger than the thermal energy. For the same particle radius given above, the charging energy is much larger than the level splitting, even if the electrostatic interaction between neighboring particles is taken into account. Therefore, $\delta E$ sets the temperature scale where the Kubo spins can be observed. However, Kubo did not take into account the possibility of intergrain electron tunneling, so that his theory strictly applies only to insulating samples. The effect of intergrain tunneling on level broadening was studied by Kawabata [2]. In addition, as studied in detail in previous works [3], a renormalization of the Coulomb blockade energy as a function of intergrain conductance is expected. The effective Coulomb blockade energy decreases exponentially with intergrain conductance and will eventually become smaller than the level splitting when intergrain coupling increases.

A review of experimental attempts at observing the Kubo spins, e.g., in indium nanoparticles, has been presented by Perenbum et al. [4,5]. Despite the compelling prediction, Kubo spins have not been confirmed experimentally yet. On the other hand, the presence of magnetic moments in weakly metallic granular Al thin films was inferred recently from magnetoresistance measurements [6] and confirmed by muon

*nimrod.bachar@unige.ch spin relaxation ( $\mu \mathrm{SR})$ spectroscopy [7]. We note that these granular Al films are superconducting at low temperatures, showing substantial intergrain electron tunneling. The exact origin of these moments coexisting with enhanced superconductivity could not be completely clarified. In addition, intergrain junction spins may also influence the $\mu$ SR response of weakly metallic films. Recent progress in utilizing granular $\mathrm{Al}$ for quantum bit (qubit) devices [8] and astrophysics photon detectors [9] requires a better understanding of the underlying physics of this system [10].

Here we present detailed and systematic $\mu$ SR measurements performed on granular Al films. The sample preparation of the series of films ranging from insulating to weakly metallic is described in Sec. II. The zero-field (ZF) $\mu$ SR measurements and analysis were performed from room temperature down to $5 \mathrm{~K}$ and described in Sec. III A. We show that the temperature dependence of the muon spin relaxation rate follows an activated behavior (Sec. III B). The activation energy becomes smaller and vanishes more quickly with temperature as we move from the insulating regime to the weakly metallic regime (Sec. III C). We argue that the value and temperature dependence (Sec. IV A) of the muon spin relaxation can be attributed to the electron's spin degree of freedom in the insulating sample, which is fully consistent with the predictions of Kubo (Sec. IV B). The behavior of the relaxation rate in the weakly metallic samples can be explained by the strongly renormalized Coulomb blockade energy (Sec. IV A), as predicted theoretically, however it requires a more fundamental understanding of the dynamic/static nature of these Kubo spins (see Sec. IVC). Finally, we conclude our results in Sec. V.

\section{SAMPLE PREPARATION}

Granular Al films were prepared by evaporation of clean (99.999\%) Al pellets in a partial pressure of oxygen. Most of the films were evaporated on $20 \mathrm{~mm}$ by $10 \mathrm{~mm}$ glass substrates that were held at liquid nitrogen temperature during the 
TABLE I. Main characteristics of samples measured in this work. The room-temperature resistivity $\rho_{\mathrm{RT}}$ was measured for each sample. $T_{c}$ was estimated from the known superconducting phase diagram of granular Al thin films [6].

\begin{tabular}{lccc}
\hline \hline Sample & $\begin{array}{c}\rho_{\mathrm{RT}} \\
(\mu \Omega \mathrm{cm})\end{array}$ & $\begin{array}{c}d \\
(\mathrm{~nm})\end{array}$ & $\begin{array}{c}T_{c} \\
(\mathrm{~K})\end{array}$ \\
\hline S140 & 140 & 2 & 2.8 \\
S380 & 380 & 2 & 3.1 \\
S1200 & 1222 & 2 & 3 \\
S1800 & 1816 & 2 & 2.9 \\
S10K & 9440 & 2 & 1.8 \\
S100K & 100000 & 3 & \\
\hline \hline
\end{tabular}

evaporation. This growth method has been proved to produce films having a narrow grain size distribution centered around $2 \mathrm{~nm}$ diam, with a nonsignificant dependence of the resistivity on size distribution for $\rho_{\mathrm{RT}}>100 \mu \Omega \mathrm{cm}[11,12]$. Some films were grown on substrates held at room temperature, resulting in $3 \mathrm{~nm}$ grains. With the increase of the partial pressure of oxygen, the insulating coating layer of the grain becomes thicker and the resistivity increases due to the lower coupling between neighboring grains [13]. Thus the surface-to-volume ratio of the grains remains rather constant while the tunneling coefficient between them can be gradually suppressed. A list of the films measured in this work and their main properties are given in Table I.

\section{EXPERIMENTAL RESULTS}

\section{A. $\mu \mathrm{SR}$ measurements and analysis}

Zero-field (ZF) $\mu \mathrm{SR}$ measurements in granular $\mathrm{Al}$ films having resistivities from 140 to $100000 \mu \Omega \mathrm{cm}$ were conducted at the low-energy muons (LEM) spectrometer at the Swiss Muon Source on the $\mu \mathrm{E} 4$ beamline [14] at the Paul Scherrer Institute (PSI) in Switzerland. In these measurements, fully polarized muons are implanted into the sample, and the time evolution of their polarization is monitored via the asymmetric beta decay [15]. The polarization of the muon ensemble, $P(t)$, is measured via detection of the emitted decay positrons as a function of time after thermalization. The time evolution of $P(t)$ is directly reflected in the asymmetry spectrum obtained from two positron detectors placed to the left and right of the sample. The implantation energy used in these measurements, typically $8 \mathrm{keV}$, was chosen based on Trim.SP Monte Carlo simulations [16] and tuned such that the muon stopping distribution has a mean depth of $55 \mathrm{~nm}$ and straggling (rms) of $13 \mathrm{~nm}$. This ensures that all the muons stop inside the film of $100 \mathrm{~nm}$ thickness. For further discussion regarding the stopping depth distribution as a function of the implantation energy, see Appendix A.

Figure 1 shows the asymmetry curves measured at selected temperatures in an insulating granular Al sample with $\rho_{\mathrm{RT}}$ of $100000 \mu \Omega \mathrm{cm}(\mathrm{S} 100 \mathrm{~K})$. The shape of the asymmetry curves is temperature-dependent. The change in shape with temperature indicates the presence of two distinct relaxation mechanisms. The first is due to the magnetic field produced by the Al nuclear moments. The second is the electronic relaxation rate, which is attributed to electronic free spins.

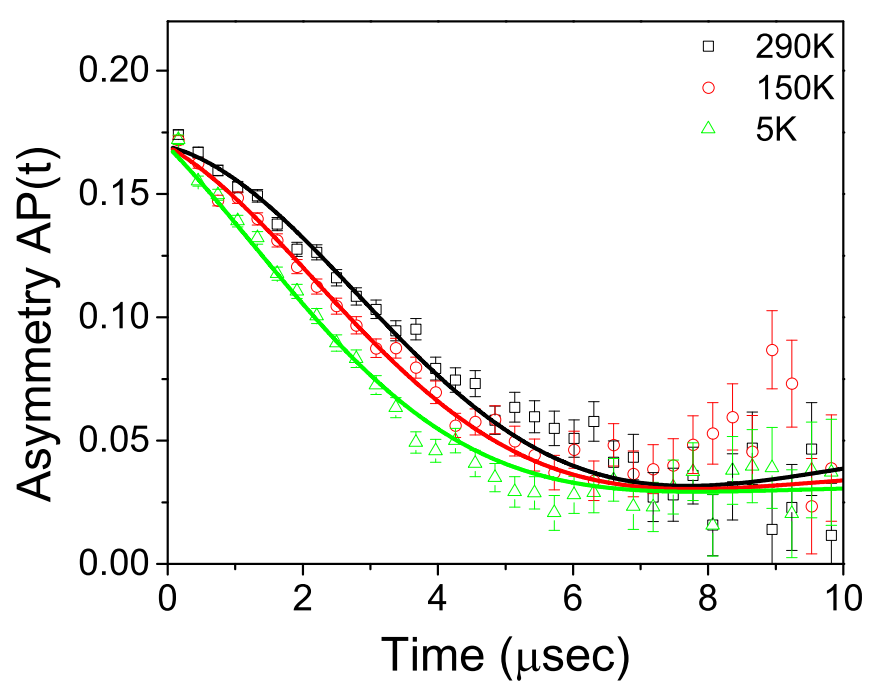

FIG. 1. Time evolution of the polarization asymmetry (symbols) at selected temperatures for insulating granular Al sample S100K with room-temperature resistivity of $100000 \mu \Omega \mathrm{cm}$ with the corresponding fit (line) according to Eq. (1). Here $A$ is a constant determined by the muon decay properties and the geometry of the $\mu$ SR spectrometer.

To account for the two relaxation processes, we use here the Kubo-Toyabe (KT) function multiplied with an exponential function $[17,18]$ :

$$
A P(t)=A_{1}\left[\frac{1}{3}+\frac{2}{3}\left(1-\sigma^{2} t^{2}\right) e^{-\frac{1}{2} \sigma^{2} t^{2}}\right] e^{-\lambda t}+A_{0},
$$

where $A_{1}$ and $A_{0}$ are the contributions of the sample and background to the measured signal, respectively. The relaxation rate $\sigma$ is the rms of the Gaussian distribution of the local magnetic fields due to the nuclear moments of $\mathrm{Al}$, and the relaxation rate $\lambda$ is attributed to fluctuating electronic magnetic moments. In the following section, we will use Eq. (1) in order to fit the experimental $\mu \mathrm{SR}$ data. As will be elaborated, we expect $\lambda$ to be temperature-dependent whereas we expect $\sigma$ to be temperature-independent. More details regarding the fit procedure are given in Appendix A.

Taking into account the contribution of each of these relaxation mechanisms, we demonstrate in Fig. 2 the expected polarization time dependence with an initial value of $P(0)=1$. In Eq. (1) with only one relaxation rate term, i.e., $\lambda / \sigma=0$, the asymmetry curve starts from its maximum value at $t=0$ with a zero slope and a negative curvature, displays a minimum at $\sigma t \sim 1.73$, and asymptotically approaches $1 / 3$ of its initial value at longer times. However, when $\lambda$ is included the time dependence of the asymmetry signal is altered from the typical $\mathrm{KT}$ form. Although the minimum in $\sigma t$ is maintained, one can see that the signal at $t=0$ already has a nearly linear drop while the long-time value is reduced dramatically. Such signatures in the asymmetry curves suggest that the contribution of the electronic relaxation rate increases.

\section{B. Insulating regime}

Figure 1 shows the corresponding fit of the modified KT function [Eq. (1)] including both relaxation rate terms, $\sigma$ and $\lambda$, to the experimental data of the insulating sample S100K. 


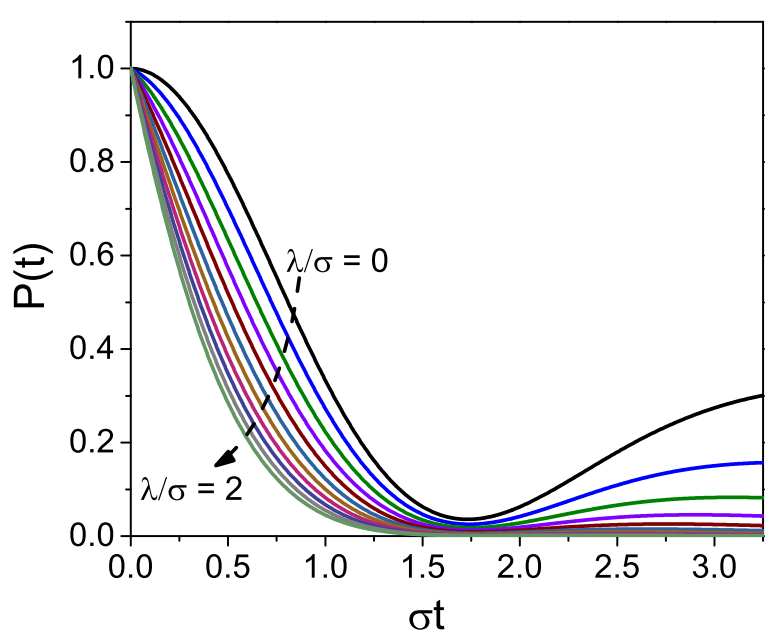

FIG. 2. Simulation of the normalized asymmetry plot using the function given in Eq. (1) of the main text. The curves were generated for the ratio of the electronic relaxation rate $\lambda$ and the nuclear relaxation rate $\sigma$ from 0 to 2 in steps of 0.2 .

As noted in the previous section, the shape of $P(t)$ changes continuously with the ratio of $\lambda / \sigma$. The crossover between the typical KT form to that described by Eq. (1) can be observed as a function of temperature in the insulating sample.

From these data it follows that at room temperature the muon spin relaxation/depolarization is dominated by the $\mathrm{Al}$ nuclear moments and that at low temperatures both electronic and nuclear moments contribute almost equally to the muon spin relaxation. Since the nuclear moments have relaxation processes much slower than the $\mu$ SR timescale, they can be considered static and temperature-independent. Therefore, the temperature dependence of $P(t)$ in the insulating sample is due to a second mechanism, which we attribute to electronic magnetic moments present in the system. The relaxation rate $\lambda$ is small at room temperature due to fast fluctuations, which slow down as temperature is lowered.

In principle, muon diffusion can also produce a temperature-dependent relaxation rate. Such a behavior has been observed in very clean $\mathrm{Al}$ samples [19]. In our films, the small grain size and the oxide coating of the Al grain acting as a barrier prohibit the muon diffusion process. Such a suppression has been observed also in previous measurements of granular and of sputtered Al films [16]. Therefore, we rule out muon diffusion as a possible source for the temperature dependency that we observe in our samples. Further discussion regarding muon diffusion is given in Appendix B.

The temperature dependence of $\lambda$ obtained from the fitted asymmetry curves for the insulating sample S100K is shown in Fig. 3. The observed temperature dependence follows at high temperature an exponential function reminiscent of a thermally activated process, whereas at low temperature it flattens. Therefore, we fit the data using a phenomenological equation, which captures the Arrhenius-type thermally activated behavior at high temperature and the saturation at low temperature:

$$
\lambda(T)=\lambda_{0}\left[1-e^{-\frac{E_{a}}{k_{B} T}}\right],
$$

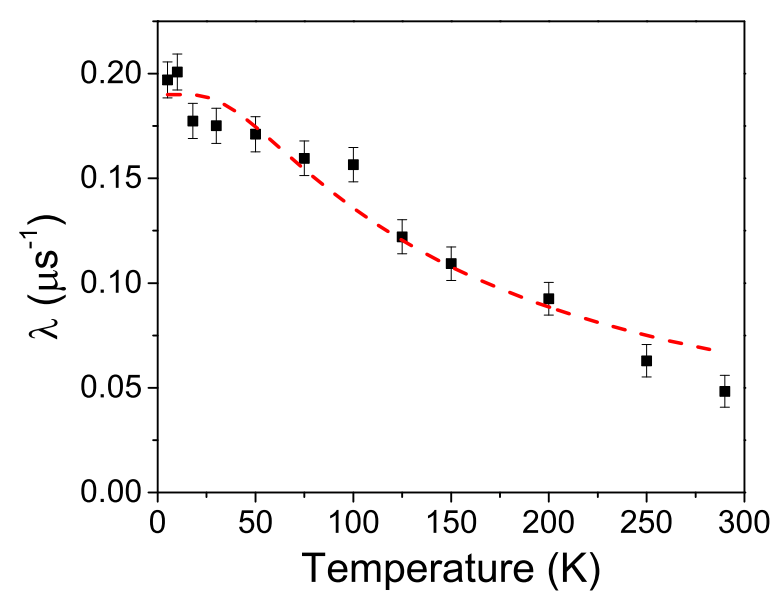

FIG. 3. Temperature dependence of the muon spin relaxation rate of electronic origin $\lambda$ for sample $\mathrm{S} 100 \mathrm{~K}$. The red dashed line follows Eq. (2) with $E_{a} \approx 125 \mathrm{~K}$ and $\lambda(0) \approx 0.19 \mu \mathrm{s}^{-1}$.

where $E_{a}$ is the activation energy and $\lambda_{0}$ is the low-temperature limit of the muon spin relaxation rate. This temperature dependence can be justified as follows: At high temperatures, all electronic spins fluctuate rapidly between up and down states due to the thermal energy and therefore do not contribute to the spin relaxation of implanted muons, i.e., $\lambda(T) \rightarrow 0$ when $k_{B} T \gg E_{a}$. As the temperature is decreased, the muon spin relaxation increases due to slowing down of thermally activated fluctuations resulting in a gradual enhancement of $\lambda(T)$. At low temperatures, $k_{B} T \ll E_{a}$, the number of activated spins saturates, resulting in the low temperature limit of the electronic depolarization rate, $\lambda(T) \rightarrow \lambda_{0}$.

\section{Metallic regime}

We have also reexamined the electronic relaxation rate in weakly metallic samples having $\rho_{\mathrm{RT}}$ in the range from 140 to about $10000 \mu \Omega \mathrm{cm}$. Selected experimental asymmetry curves along with their fit to Eq. (1) for all samples are shown in Fig. 4.

From the fit, and following our previous discussion, we have extracted the values of $\lambda$ as a function of temperature for these samples (Fig. 5). At first glance they seem to overlap, but a closer examination reveals that there are differences among these metallic samples, particularly at high temperatures. While for all samples $\lambda$ decreases as the temperature is increased, in the highest resistivity one (S10K) it is still substantial at room temperature while for a lower resistivity one (S380) it is barely measurable. The value of the activation energy obtained from fits to Eq. (2) goes down progressively as the resistivity decreases. For sample S10K the activation temperature is still close to the value of the insulating sample, but it is definitely lower in the metallic regime and falls down to $30-50 \mathrm{~K}$ for the most metallic samples (see Table II).

\section{DISCUSSION}

\section{A. Level splitting and Coulomb energy in nanometallic grains}

In the previous section, we used the temperature dependence of the electronic relaxation rate $\lambda$ to extract an activation 


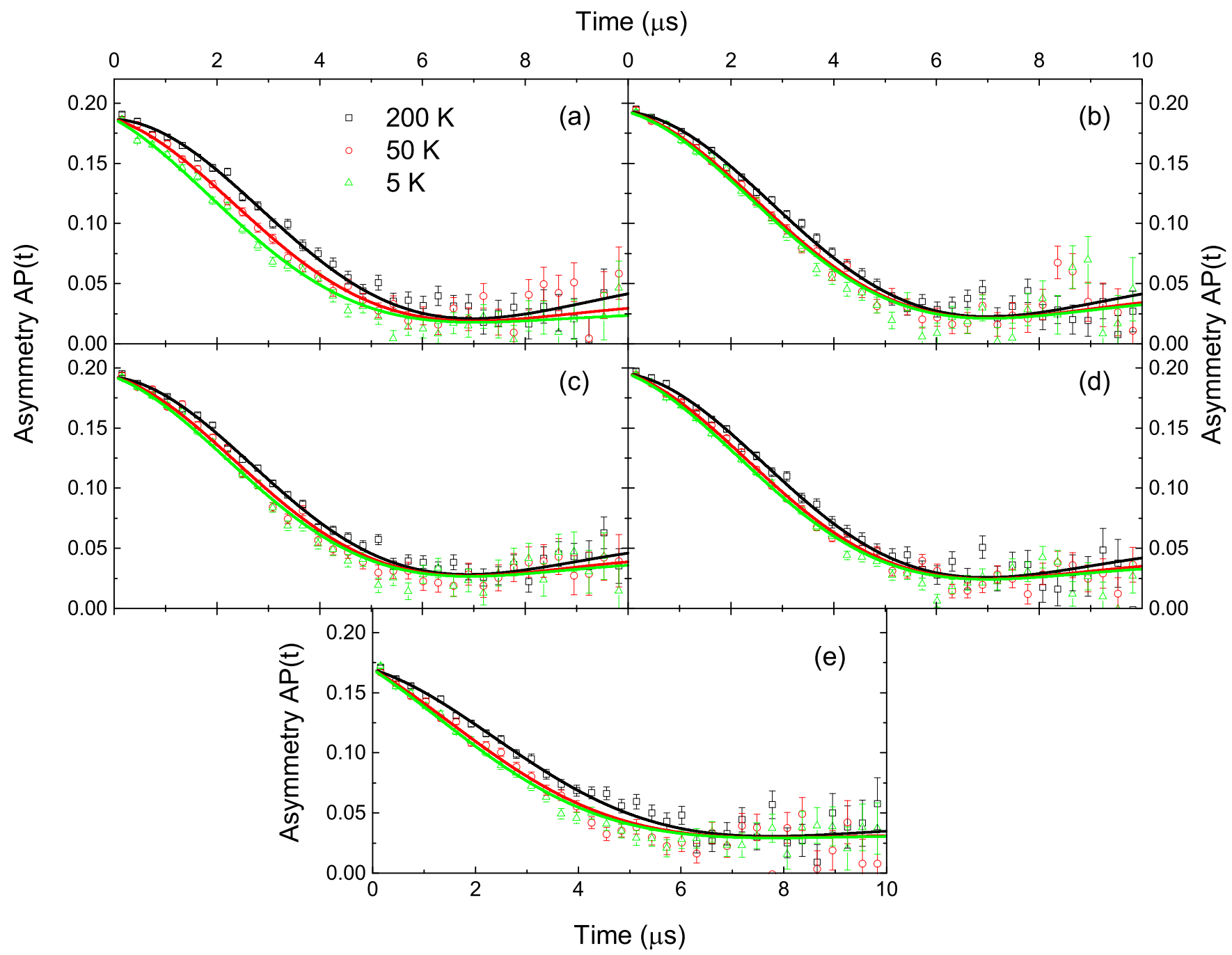

FIG. 4. Summary of asymmetry curves at selected temperatures for samples measured in this work (see Table I): (a) $380 \mu \Omega \mathrm{cm}$, (b) $1222 \mu \Omega \mathrm{cm}$, (c) $1816 \mu \Omega \mathrm{cm}$, (d) $9440 \mu \Omega \mathrm{cm}$, and (e) $100000 \mu \Omega \mathrm{cm}$. Open symbols represent the experimental data and full lines represent fits to Eq. (1). The insulating sample data are presented now at the same temperatures as those of the metallic/superconducting samples.

energy $E_{a}$. The fit to Eq. (2) on the insulating sample gives an activation energy $E_{a}$ of about $10 \mathrm{meV}$ or equivalently an activation temperature of about $120 \mathrm{~K}$ (see Table II). This value is much smaller than the electrostatic charging energy, which is $80 \mathrm{meV}$ for isolated grains and estimated to be $30 \mathrm{meV}$ when taking into account the electrostatic interaction between neighboring grains [20]. Since in this insulating sample the electrons are localized, there is no renormalization of the charging energy due to intergrain electron tunneling. On the other hand, the average electronic level splitting due to quantum effects is defined by the grain size, and coupling to other grains (or thermal energy) will only smear the discreteness of these electronic levels. We therefore ascribe the measured activation energy to the average energy level splitting rather than to the charging energy. Taking into account that the Fermi energy of $\mathrm{Al}$ is $11.7 \mathrm{eV}$ and a level spacing of $\delta E=10 \mathrm{meV}$, we have thus about 1170 electronic levels per grain. Assuming the free electron model without the spin weight [1] where $E_{F} / \delta E=3 Z N / 4$ and that each $\mathrm{Al}$ atom contributes three electrons, i.e., $Z=3$, we have $N=520 \mathrm{Al}$ atoms per grain, which gives a spherical grain diameter of $2.5 \mathrm{~nm}$. This is in excellent agreement with the average grain size of $3 \mathrm{~nm}$ measured in high resistivity Al granular films [11].

On the other hand, the low activation energy in the metallic regime can probably be ascribed to the strongly renormalized electrostatic charging energy, rather than the energy level splitting. This renormalization is known to decrease exponentially the charging energy of a quantum dot as a function of the conductance of the contacts coupling it to two bulk leads. For weak contacts it reads as [3]

$$
E_{C}^{*}=2 \pi \alpha_{t} E_{C} e^{-\pi^{2} \alpha_{t}},
$$

where $\alpha_{t}$ is the bare conductance of the contacts normalized to the universal conductance $G=e^{2} / h$. From the roomtemperature resistivity of the insulating sample and the grain size of $3 \mathrm{~nm}$, the bare intergrain conductance is of the order of $1 \times 10^{-6} \Omega^{-1}$ and the renormalization effect is small. For the metallic samples, the bare conductance of the intergrain contact is of the same order as the universal conductance, and the renormalization effect is large. At some point the effective 


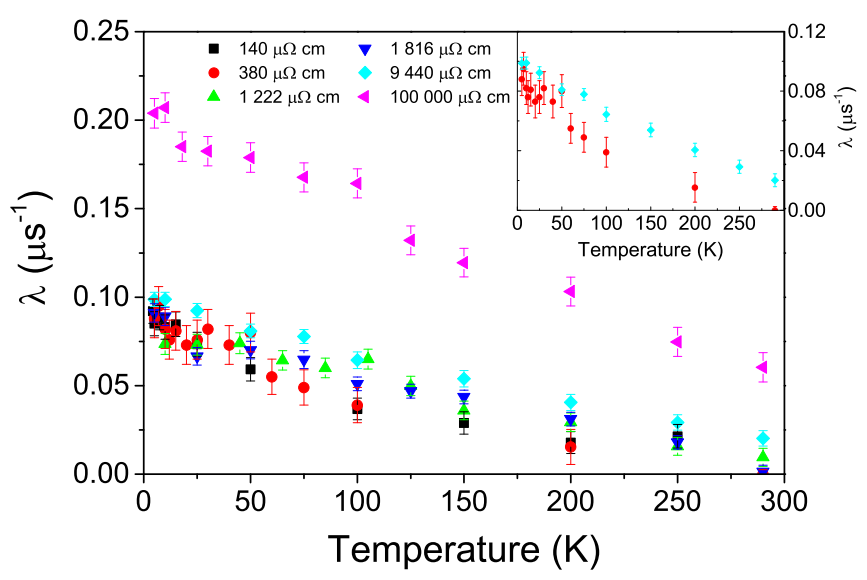

FIG. 5. Temperature dependence of the muon spin relaxation rate of electronic origin, $\lambda$, for all samples in this work. The inset shows a comparison of $\lambda$ between samples S380 and S10K.

charging energy becomes smaller than the level splitting and becomes the dominant energy scale for the observation of the Kubo spin. This seems to occur close to the metal-to-insulator transition [21] when $\rho_{\mathrm{RT}}$ is about $10000 \mu \Omega \mathrm{cm}$.

It is interesting to compare the activation energy scale obtained by $\mu \mathrm{SR}$ in the metallic samples to that of the multilevel Kondo effect obtained from transport measurements. When the renormalized charging energy is smaller than the level splitting, the conductivity of a nanodot is predicted to start out metallic-like at high temperatures, increasing as the temperature goes down, and to become insulating-like when the temperature reaches the renormalized charging energy [3]. This behavior was indeed observed in granular $\mathrm{Al}$ films having resistivities of $200-300 \mu \Omega \mathrm{cm}$, with the downturn of the conductivity occurring at about $50 \mathrm{~K}$ [22]. It is remarkable that this is indeed the value of the activation energy that we obtain from $\mu \mathrm{SR}$ for similar resistivity values.

\section{B. Spin concentration}

As can be seen from Fig. 5, the relaxation rate $\lambda$ saturates at low temperatures to a value of about $0.09 \mu \mathrm{s}^{-1}$ for the metallic samples, while it reaches a value of $0.197(9) \mu \mathrm{s}^{-1}$ in the insulating sample.
From the values of the electronic relaxation rate at low temperatures, we can extract the estimated concentration of free spins [7]. In zero field, the relaxation rate due to the nuclear spins of ${ }^{27} \mathrm{Al}\left(I_{N}=5 / 2,100 \%\right.$ abundance $)$ is given by [23-25]

$$
\sigma^{2}=\frac{2}{3}\left(\frac{\mu_{0}}{4 \pi}\right)^{2} \hbar^{2} \gamma_{\mu}^{2} \gamma_{N}^{2} I_{N}\left(I_{N}+1\right) \sum_{i} \frac{1}{r_{i}^{6}} .
$$

The exponential relaxation rate $\lambda$ corresponds to a spin concentration of

$$
c=\frac{1}{1.18} \frac{\lambda}{\sigma_{\max }},
$$

where the numerical factor takes into account the ratio between the full width at half-maximum of a Gaussian distribution and that of a Lorentzian distribution of fields (as reflected by the exponential relaxation). In the case of a ${ }^{27} \mathrm{Al}$ nuclear moment with an additional $1 \mu_{B}$ electronic moment of spin $1 / 2$, the rate would be a factor $\sqrt{\left[\gamma_{e}^{2} s(s+1)\right] /\left[\gamma_{\mathrm{Al}}^{2} I_{N}\left(I_{N}+1\right)\right]}$ higher, where $\gamma_{e}$ and $\gamma_{\mathrm{Al}}$ are the electronic and nuclear gyromagnetic ratio, receptively. Therefore, $\sigma_{\max } \equiv 186 \mu \mathrm{s}^{-1}$.

For the metallic samples ranging from 140 to about $10000 \mu \Omega \mathrm{cm}$, we obtained a relaxation rate value of $\lambda \approx$ $0.09 \mu \mathrm{s}^{-1}$, which implies a free spins concentration of about $400 \mathrm{ppm}$. Considering the estimation that each grain holds about $520 \mathrm{Al}$ atoms, we obtain a spin concentration of about one spin per four to five grains [7].

The value of $\lambda \approx 0.197 \mu \mathrm{s}^{-1}$ for the insulating sample implies a spin concentration of about $860 \mathrm{ppm}$, or one spin per two grains. We reach the conclusion that there is on the order of one Kubo spin for two grains, as expected in the case of isolated finite-size metallic grains [1].

We note here that Kubo spins are only present in grains where electrons are localized as in the insulating sample. The coexistence of localized and delocalized electrons in high resistivity metallic samples is known and leads to percolation effects in the superconducting state [26].

In addition to the Kubo spin, which is a volume effect, a surface effect may also be at the origin of magnetic moments [7]. Since the oxide coating of the grain is not perfect, dangling oxygen bonds or adsorbed molecules on its surface $[27,28]$ can result in a surface spin density. A para- to antiferromagnetic transition of solid $(\gamma-\beta)$ oxygen

TABLE II. Summary of the fit values of the experimental data to the modified Kubo-Toyabe function [Eq. (1)] with a nuclear spin relaxation rate (depicted by $\sigma$ ) and an electronic spin relaxation rate (depicted by $\lambda$ ) along with a background bias signal (depicted by $A_{0}$ ). The uncertainty in these values is given in parentheses. The $\alpha$ values have no uncertainty as they were estimated from separated calibration measurements and kept fixed in the fit to the ZF data. The quality of the fit is given by the reduced $\chi^{2}$ value, which was normalized to the number of degrees of freedom (NDF) of the model. The activation temperature $E_{a}$ was obtained from the muon spin relaxation rate $\lambda$ by fitting all experimental

\begin{tabular}{|c|c|c|c|c|c|c|c|}
\hline $\mathrm{S} 140$ & 140 & 0.9474 & $0.11811(0.0008)$ & $0.2478(0.003)$ & $0.02237(0.0006)$ & 1.035 & $55(4)$ \\
\hline S1200 & 1222 & 0.9977 & $0.17542(0.0008)$ & $0.2481(0.002)$ & $0.01727(0.0007)$ & 1.021 & $108(15)$ \\
\hline S1800 & 1816 & 1.0149 & $0.16901(0.0007)$ & $0.2525(0.002)$ & $0.02346(0.0006)$ & 1.015 & 88 (16) \\
\hline S10K & 9440 & 1.038 & $0.17395(0.0008)$ & $0.2502(0.002)$ & $0.02108(0.0007)$ & 1.026 & $102(8)$ \\
\hline
\end{tabular}
temperature data to Eq. (2). 
at a temperature of $43 \mathrm{~K}$ is observed in the magnetic susceptibility of adsorbed oxygen on, e.g., a graphite surface [29]. Surface spins might be one of the main causes for the phase decoherence effect in qubit devices fabricated using an $\mathrm{Al}$ and $\mathrm{Nb}$ superconducting quantum interference device (SQUID). Such spins were measured indirectly by the quantum flux noise $1 / f$ in qubit devices [30,31] and directly by scanning SQUID magnetometry [32]. The experimental surface spin density was found to be about $4 \times 10^{17} \mathrm{~m}^{-2}$. Recent theoretical works suggested that the origin of the $1 / f$ flux noise is due to either strong coupling of free spins mediated by the Ruderman-Kittel-Kasuya-Yosida interaction from conduction electrons [33], or to weak anisotropic dipole-dipole coupling of spin clusters [34]. The latter was shown to explain the temperature dependence and geometry independence of the $1 / f$ noise by taking into account the experimental surface spin density value of $4 \times 10^{17} \mathrm{~m}^{-2}$.

However, the temperature dependence of $\lambda$ that we observe cannot be easily explained by surface spins. Surface spins cannot hop between different grains and are not expected to exhibit an activated temperature dependence, unlike the Kubo spins, which only appear at low temperatures when electrons become localized in each grain. We therefore propose that the volume Kubo spin rather than surface spins is at the origin of magnetic moments in the nanosized Al grains. Nevertheless, we cannot rule out completely that in the strong grain coupling regime (low $\rho_{\mathrm{RT}}$ ), surface spins presumably located at the oxide interface between the grains could play a role in the temperature dependence of the muon relaxation rate around the oxygen AF transition.

\section{Dynamic versus static behavior of Kubo spins}

Until now we have analyzed the data using a model that takes into account two relaxation rates: The first is a static and temperature-independent relaxation rate, $\sigma$, which we ascribed to the nuclear moments. The second is a temperaturedependent relaxation rate $\lambda$ attributed to electronic spins, in particular Kubo spins. We observed an activation behavior in $\lambda$ that can be understood in the following way: as temperature is lowered, the electronic relaxation rate is enhanced due to an increasing concentration of thermally activated Kubo spins.

A different scenario suggests that the temperature dependence of the muon spin relaxation rate that we show in our data can be understood as a change in the static versus dynamic relaxation rates due to electronic spins becoming static at low temperatures while being dynamic at higher temperatures. To address this question, we have fitted our data to Eq. (1) allowing now both $\sigma$ and $\lambda$ to be temperaturedependent in our analysis. Figure 6 shows the result of such analysis for the insulating sample S100K and sample S10K, which is close to the metal-to-insulator transition. Although we allow $\sigma$ to change as a function of temperature, we still detect a strong enhancement in the value of $\lambda$. Here $\sigma$ changes from a value of about $0.2 \mu \mathrm{s}^{-1}$ at high temperatures to a value of about $0.25 \mu \mathrm{s}^{-1}$ at low temperatures. At the same time, $\lambda$ doubles its value at low temperatures compared to its value at room temperature. The same analysis in samples on the metallic side results in less pronounced changes in $\lambda(T)$ except for sample S10K, which is shown in Fig. 6.

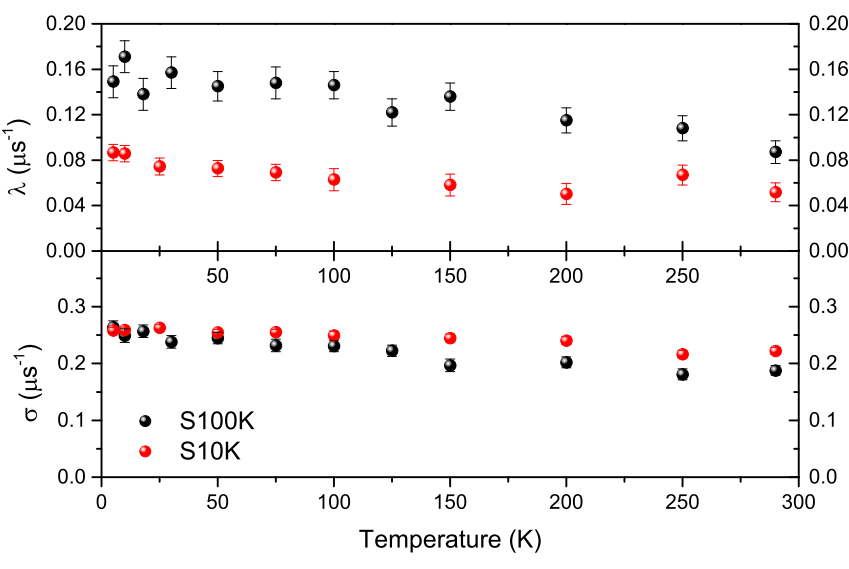

FIG. 6. Temperature dependence of the muon spin relaxation rates $\lambda$ and $\sigma$ for samples $\mathrm{S} 10 \mathrm{~K}$ and $\mathrm{S} 100 \mathrm{~K}$.

In the case of the insulating sample, a strong temperature dependence in the electronic relaxation rate is seen in both kinds of analysis, i.e., the first with only $\lambda(T)$ and the second which adds also $\sigma(T)$. This confirms that the Kubo spins that are of electronic origin and are thermally activated when $k_{B} T \ll \delta E$ are the main source for the local moments that we detect in the insulating sample. However, in the metallic samples, this temperature dependence is susceptible to the chosen model. Therefore, it is possible that the metallic regime, where the coexistence of localized and delocalized electrons is present, requires additional longitudinal field measurements and a more complicated fitting function.

With our current data and analysis, we cannot confirm the static nature of these Kubo spins and the crossover from a static behavior at low temperatures to a dynamic behavior at high temperatures as a function of the grain coupling factor. Therefore, longitudinal field (LF) -dependent measurements are required on films ranging from weakly metallic to insulating. Here a full magnetic-field-temperature mapping could reveal the true static nature of these localized moments and its correlation with the activation energy that we have obtained. We expect that in the insulating regime, the Kubo spins will be fully static below the activation energy, while in the metallic regime this crossover will be influenced by the interplay between the renormalized Coulomb energy $E_{C}^{*}$ and the energy level splitting $\delta E$.

\section{CONCLUSIONS}

In conclusion, the $\mu \mathrm{SR}$ results on granular Al films presented and analyzed here provide direct experimental evidence of the Kubo spin effect. As expected, this evidence is most compelling in the insulating regime. The activation energy obtained from the temperature dependence of the electronic relaxation rate, $125 \mathrm{~K}$, is in excellent agreement with the average energy level splitting in an ensemble of metallic particles, and the number of spins per grain is of the order of one for a couple of grains. The value of the activation energy in metallic samples is reduced and is in agreement with the effective charging energy obtained from a multilevel Kondo analysis of the transport data for these films. However, 


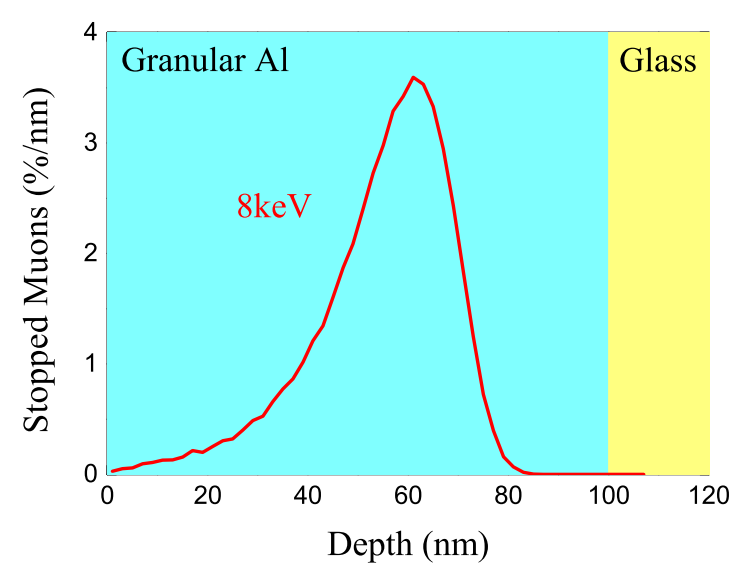

(a)

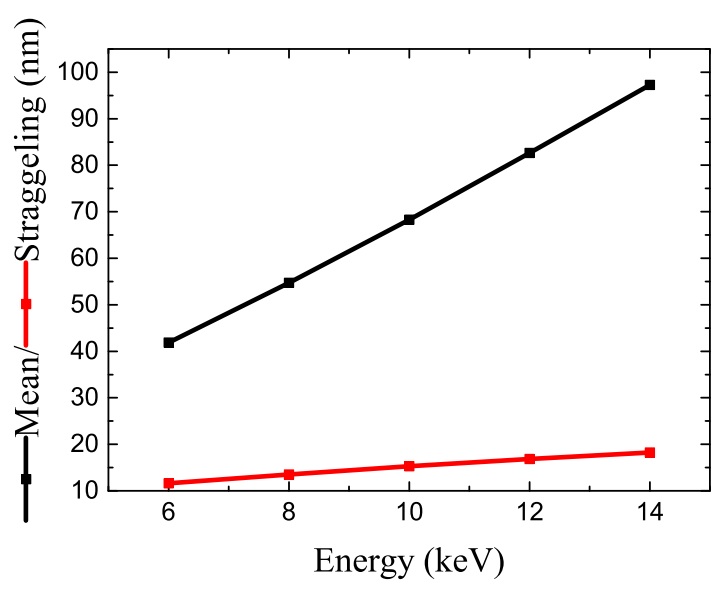

(b)

FIG. 7. (a) Trim.SP Monte Carlo simulations [16] of the muon stopping distribution in a $100 \mathrm{~nm}$ thick granular Al sample where the implantation energy is $8 \mathrm{keV}$. The distribution has a mean depth value of $55 \mathrm{~nm}$ with straggling of $13 \mathrm{~nm}$. (b) The mean depth and straggling values of the muon stopping distribution as a function of the implantation energy.

future LF measurements should be done in order to unravel the dynamics of these Kubo spins as a function of temperature.

\section{ACKNOWLEDGMENTS}

We would like to thank Amit Kanigel, Antoine Georges and Serge Florens for useful discussions. This work is based on experiments performed at the Swiss Muon Source $(\mathrm{S} \mu \mathrm{S})$, Paul Scherrer Institute (PSI), Villigen, Switzerland.

\section{APPENDIX A: MUON SPIN RELAXATION}

The muons were implanted at a typical energy of $8 \mathrm{keV}$ except for sample S140, where $10 \mathrm{keV}$ energy was used. Figure 7 shows the stopping distribution using Trim.SP Monte Carlo simulations. We note here that the simulations were done taking into account bulk aluminum density while the effective medium of our films is composed of metallic $\mathrm{Al}$ grains and insulating amorphous $\mathrm{Al}_{2} \mathrm{O}_{3}$ matrix. This could slightly modify the actual implantation profile. However, calibration measurements as a function of depth and in various films show a negligible difference in the signal at 6 and $8 \mathrm{keV}$. Only at $10 \mathrm{keV}$ is a smaller $\mu \mathrm{SR}$ signal detected, probably due to a fraction of muons stopping in the substrate and forming muonium.

The muon spin relaxation data were collected while cooling down the sample under zero magnetic field and with a muon spin rotation angle of $-10^{\circ}$ with respect to the direction to the left detector. The asymmetry data are obtained from the left and right set of detectors.

Before each temperature scan and for each sample, we performed a measurement in transverse field of $B=50 \mathrm{G}$ and at room temperature in order to obtain the factor $\alpha$, which takes into account possible differences in the solid angle and efficiency between the detectors used to determine the asymmetry as well as beam misalignment to be used for fitting the zero-field measurements. We find $\alpha$ close to 1 as expected in ideal conditions. The value of $\alpha$ was fixed in the following zero-field fit.
The time evolution of the asymmetry curve can be described by the modified KT equation [Eq. (1)]. As was elaborated, it includes two terms to describe the muon spin relaxation rate: The first is the nuclear relaxation rate $\sigma$, which is due to the Al nuclear moments and is assumed to be temperature-independent. The second is the electronic relaxation rate $\lambda$, which is attributed to electronic free spins in the sample and can show temperature dependency.

We fit all zero-field (ZF) data for one sample obtained during one cool down with the modified KT function using the musrfit package [35]. The asymmetry data were fitted from $t=0.07 \mu \mathrm{s}$ in order to exclude the short-time decay rate coming from muons stopping in the $\mathrm{Ni}$ backing. We note that the asymmetries that we used for the fits were generated using the post-pileup corrected (PPC) histograms to accurately determine the uncorrelated background contribution in the histograms. We used a longer time window of up to $t=10 \mu \mathrm{s}$ in our fit, and we did not detect a change in the extracted values of $\lambda$ compared to a fit in a shorter time window of $t=8 \mu \mathrm{s}$. This is due to the fact that the fit values are dominated by the exponential decay at intermediate times where the error bars are relatively small (and since sigma is temperature-independent). As described in the main text, this exponential decay is associated with the electronic relaxation rate $\lambda$, which is temperature-dependent in our samples.

The parameters used for the fit as given by Eq. (1) are the relaxation rates $\sigma$ and $\lambda$, the initial asymmetry value at zero time for the sample, $A_{1}$, and the calibration parameter between the left and right detectors, $\alpha$. The parameter $A_{0}$ takes into account the contribution of the 1/3 tail from muons stopping in the Ni backing, which is nonrelaxing, and the glass substrate, which is also nonrelaxing, due to the absence of any nuclear or electronic moments. All mentioned parameters except $\lambda$ were shared in our fit procedure resulting in common values for the entire temperature scan of each sample. The obtained fit values are summarized in Table II for all samples in this work. The initial asymmetry was found to be $A_{1} \approx 0.17$ in most of the samples except two. In the most metallic sample S140 a muon implantation energy of $10 \mathrm{keV}$ was used instead 
of the typical $8 \mathrm{keV}$, which therefore resulted in a lower signal from the film as muons stop deeper into the glass substrate and lose part of their polarization due to muonium formation. In the most insulating sample $\mathrm{S} 100 \mathrm{~K}$ a reduction of the signal should be expected due to the higher insulator-to-metal ratio. We note that although $A_{1}$ was found to be lower in these two samples, the value of $\sigma$ that was obtained from the fit represents the crossover from the metallic regime to the insulating regime. A typical value of $\sigma \approx 0.25 \pm 0.01 \mu \mathrm{s}^{-1}$ was shown to be resistivity-independent in the metallic regime (where we expect the $\mathrm{Al}: \mathrm{Al}_{2} \mathrm{O}_{3}$ volume fraction to be of the order of $0.8[11-13,36])$ while only at the insulating sample (where the $\mathrm{Al}: \mathrm{Al}_{2} \mathrm{O}_{3}$ volume fraction is already below 0.5 ) did this value change to $\sigma \approx 0.22 \mu \mathrm{s}^{-1}$. Such values are in between the expected values for pure $\mathrm{Al}\left(0.3 \mu \mathrm{s}^{-1}\right)$ and $\mathrm{Al}$ oxide $\left(0.2 \mu \mathrm{s}^{-1}\right)$ [7], reflecting a volume weighted average of the two contributions. From the above fit procedure, we finally deduced the temperature-dependent value of $\lambda(T)$ as shown in Fig. 5.

\section{APPENDIX B: MECHANISMS FOR THE TEMPERATURE-DEPENDENT DEPOLARIZATION RATE}

In principle, temperature-dependent muon diffusion can also induce a temperature dependence of the depolarization rate due to the muon probing a different nuclear moments environment. In the past, this diffusion was studied down to the lowest temperatures $(30 \mathrm{mK})$ and was found to take place in very pure $\mathrm{Al}$ with less than $1 \mathrm{ppm}$ overall impurities and RRR $>10000$. Upon adding impurity atoms in the few to $>1000$ ppm level (see, e.g., Ref. [19]), due to trapping-detrapping processes a nonmonotonic relaxation rate versus temperature behavior was found with maxima of the relaxation rate around $10-50 \mathrm{~K}$. The maxima correspond to the muon being static. The trapping rate was found by Kehr et al. [19] to be proportional to the impurity concentration.

By contrast, our samples consist of very small $\mathrm{Al}$ grains (about $2 \mathrm{~nm}$ ) with a solid oxide barrier between them, which will impede the muon diffusion. For a rough estimate of the equivalent impurity level in nanograin $\mathrm{Al}$, we assumed an oxide layer on a $2 \mathrm{~nm} \mathrm{Al}$ sphere. This gives about $50000 \mathrm{ppm}$ impurity concentration. The effective number of trapping centers can be even higher than that because an impurity can produce several (up to 100) trapping centers. Taking the trapping rates from Kehr et al., we get a trapping time of $20 \mathrm{ns,}$ which is even an upper limit so that we can safely assume that muon diffusion does not influence our results.

Therefore, muon diffusion can be neglected, and the temperature-independent Kubo-Toyabe rate $\sigma \simeq 0.25 \mu \mathrm{s}^{-1}$ that we observe corresponds to a static muon depolarization due to the nuclear moments in $\mathrm{Al}$ and its oxide, whereas the monotonous exponential rate is of an electronic origin.
[1] R. Kubo, J. Phys. Soc. Jpn. 17, 975 (1962).

[2] A. Kawabata, J. Phys. Soc. Jpn. 43, 1491 (1977).

[3] S. Florens, P. San José, F. Guinea, and A. Georges, Phys. Rev. B 68, 245311 (2003).

[4] J. Perenboom, P. Wyder, and F. Meier, Phys. Rep. 78, 173 (1981).

[5] J. A. A. J. Perenboom, P. Wyder, and F. Meier, Phys. Rev. B 23, 279 (1981)

[6] N. Bachar, S. Lerer, S. Hacohen-Gourgy, B. Almog, and G. Deutscher, Phys. Rev. B 87, 214512 (2013).

[7] N. Bachar, S. Lerer, A. Levy, S. Hacohen-Gourgy, B. Almog, H. Saadaoui, Z. Salman, E. Morenzoni, and G. Deutscher, Phys. Rev. B 91, 041123(R) (2015).

[8] N. Maleeva, L. Grünhaupt, T. Klein, F. Levy-Bertrand, O. Dupré, M. Calvo, F. Valenti, P. Winkel, F. Friedrich, W. Wernsdorfer, A. V. Ustinov, H. Rotzinger, A. Monfardini, M. V. Fistul, and I. M. Pop, Nat. Commun. 9, 3889 (2018).

[9] O. Dupré, A. Benoît, M. Calvo, A. Catalano, J. Goupy, C. Hoarau, T. Klein, K. L. Calvez, B. Sacépé, A. Monfardini, and F. Levy-Bertrand, Supercond. Sci. Technol. 30, 045007 (2017).

[10] L. Grünhaupt, N. Maleeva, S. T. Skacel, M. Calvo, F. LevyBertrand, A. V. Ustinov, H. Rotzinger, A. Monfardini, G. Catelani, and I. M. Pop, Phys. Rev. Lett. 121, 117001 (2018).

[11] G. Deutscher, H. Fenichel, M. Gershenson, E. Grünbaum, and Z. Ovadyahu, in Low Temperature Physics-LT 13, Vol. 3, edited by K. D. Timmerhaus, W. J. O'Sullivan, and E. F. Hammel (Springer, Boston, MA, 1974), pp. 573-577; J. Low Temp. Phys. 10, 231 (1973).
[12] G. Deutscher, M. Gershenson, E. Grünbaum, and Y. Imry, J. Vac. Sci. Technol. 10, 697 (1973).

[13] Y. Shapira, G. Deutscher, and T. Suv, Thin Solid Films 87, 29 (1982).

[14] T. Prokscha, E. Morenzoni, K. Deiters, F. Foroughi, D. George, R. Kobler, A. Suter, and V. Vrankovic, Nucl. Instrum. Methods Phys. Res. Sect. A 595, 317 (2008).

[15] E. Morenzoni, H. Glückler, T. Prokscha, H. Weber, E. Forgan, T. Jackson, H. Luetkens, C. Niedermayer, M. Pleines, M. Birke, A. Hofer, J. Litterst, T. Riseman, and G. Schatz, Physica B 289290, 653 (2000).

[16] E. Morenzoni, H. Glückler, T. Prokscha, R. Khasanov, H. Luetkens, M. Birke, E. Forgan, C. Niedermayer, and M. Pleines, Nucl. Instrum. Methods Phys. Res. Sect. B 192, 254 (2002).

[17] R. S. Hayano, Y. J. Uemura, J. Imazato, N. Nishida, T. Yamazaki, and R. Kubo, Phys. Rev. B 20, 850 (1979).

[18] Y. J. Uemura, T. Yamazaki, D. R. Harshman, M. Senba, and E. J. Ansaldo, Phys. Rev. B 31, 546 (1985).

[19] K. W. Kehr, D. Richter, J. M. Welter, O. Hartmann, E. Karlsson, L. O. Norlin, T. O. Niinikoski, and A. Yaouanc, Phys. Rev. B 26, 567 (1982).

[20] B. Abeles, Phys. Rev. B 15, 2828 (1977).

[21] M. Kunchur, P. Lindenfeld, W. L. McLean, and J. S. Brooks, Phys. Rev. Lett. 59, 1232 (1987).

[22] A. Moshe, N. Bachar, S. Lerer, Y. Lereah, and G. Deutscher, J. Supercond. Nov. Magn. 31, 733 (2018).

[23] J. H. Van Vleck, Phys. Rev. 74, 1168 (1948). 
[24] O. Hartmann, Phys. Rev. Lett. 39, 832 (1977).

[25] P. D. de Réotier and A. Yaouanc, J. Phys.: Condens. Matter 9, 9113 (1997).

[26] G. Deutscher, O. Entin-Wohlman, S. Fishman, and Y. Shapira, Phys. Rev. B 21, 5041 (1980).

[27] D. Lee, J. L. DuBois, and V. Lordi, Phys. Rev. Lett. 112, 017001 (2014).

[28] H. Wang, C. Shi, J. Hu, S. Han, C. C. Yu, and R. Q. Wu, Phys. Rev. Lett. 115, 077002 (2015).

[29] S. Gregory, Phys. Rev. Lett. 40, 723 (1978).

[30] S. Sendelbach, D. Hover, A. Kittel, M. Mück, J. M. Martinis, and R. McDermott, Phys. Rev. Lett. 100, 227006 (2008).
[31] S. M. Anton, J. S. Birenbaum, S. R. O'Kelley, V. Bolkhovsky, D. A. Braje, G. Fitch, M. Neeley, G. C. Hilton, H.-M. Cho, K. D. Irwin, F. C. Wellstood, W. D. Oliver, A. Shnirman, and J. Clarke, Phys. Rev. Lett. 110, 147002 (2013).

[32] H. Bluhm, J. A. Bert, N. C. Koshnick, M. E. Huber, and K. A. Moler, Phys. Rev. Lett. 103, 026805 (2009).

[33] L. Faoro and L. B. Ioffe, Phys. Rev. Lett. 100, 227005 (2008).

[34] J. Atalaya, J. Clarke, G. Schön, and A. Shnirman, Phys. Rev. B 90, 014206 (2014).

[35] A. Suter and B. M. Wojek, Phys. Procedia 30, 69 (2012).

[36] P. Ziemann, G. Heim, and W. Buckel, Solid State Commun. 27, 1131 (1978). 